\title{
Challenges for meaningful interpersonal communication in a digital era
}

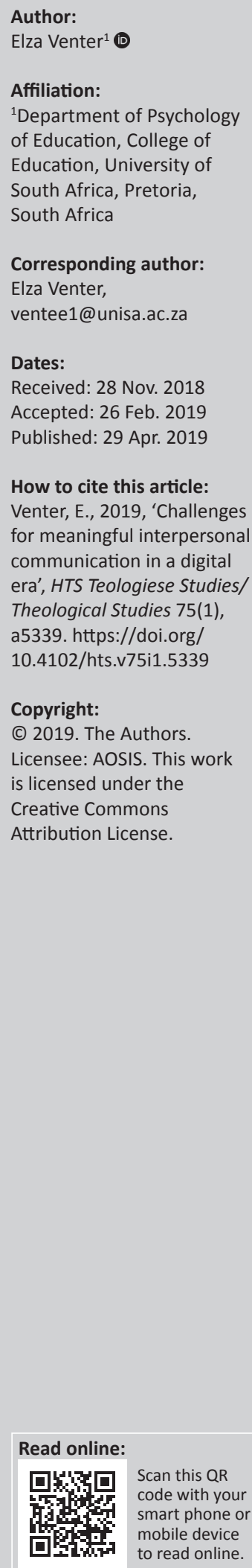

As digital tools and social networks became the main mode of interaction for many people, interpersonal communication has changed. Computer-mediated communication (CMC) has become more important than face-to-face communication in many contexts. Younger generations prefer CMC. Personal interaction normally consists of verbal and non-verbal communication. Computer-mediated communication lacks traditional non-verbal cues, which may cause misunderstandings, influencing meaningful interpersonal communication. Because of a lack of face-to-face communication, people often present an idealised version of themselves, thus becoming less inhibited involving more inappropriate self-disclosure on, for instance, social networks. The research question for this literature review was whether communicating with others mainly through digital means without adequate non-verbal cues would influence meaningful interaction between people. This study used the cues-filtered-out approach and the social presence theory with a literature review to get some clarity on the above question. The premise of the author was that because of the lack of non-verbal cues, CMC messages could influence the understanding of emotions and attitudes, thus compromising meaningful communication and personal understanding of the other. The outcome was that in today's world people have to use $\mathrm{CMC}$, but for meaningful interpersonal communication, they should try to combine it with face-to-face interaction.

Keywords: Face-to-face communication; Computer-mediated communication; Verbal communication; Non-verbal communication; Meaningful interpersonal communication; Self-disclosure; De-individuation.

\section{Introduction}

The word 'communication' is derived from the Latin word 'communis', meaning to share. Communication indicates the sharing of words, emotions, ideas, intent and messages (Alshenqeeti 2016:58; Lamichhane 2016:91) between human beings. It is an ongoing process, not only to convey ideas and information but also to try to make meaning together. Good communication is an art that should be developed (Lamichhane 2016:91). It is an everyday activity at 'the core of all human contact' fundamental to human life. It helps us to build relationships with others through mutual understanding. No individual or social system can function without communication (Ayee 2013:4-5). Communication, thus, makes us human. It is an interactive process where people try to understand each other (Hartman \& McCambridge 2011:28) in order to co-exist in a meaningful way. When either verbal or non-verbal communication is lacking, meaningful communication could be a challenge.

Since digital communication tools and social networks became such an important way of communication, especially for younger generations, interpersonal interaction has changed. Interpersonal communication consists of face-to-face interactions as well as computer-mediated communication (CMC) in this digital world (Drago 2015:13). Face-to-face interaction consists of both verbal and non-verbal communication where two or more people use words, gestures or body language to convey their message. Face-to-face communication helps clarify meaning to avoid misunderstandings between people, because of verbal and non-verbal cues. Interpersonal communication, however, can also occur through CMC via the use of digital tools or social networks. These tools assist in creating a personal connection through talking on a phone, exchanging text or instant messages, or using social network sites to share information (Solomon \& Theiss 2013:6; Venter 2017:497), although more through verbal than non-verbal cues.

The availability of the internet and interactive communication technologies became a way of communicating simultaneously with many people (Jiang, Bazarova \& Hancock 2010:58). The

Note: SASRF 2019, sub-edited by Cornel du Toit (University of South Africa) and Wessel Bentley (University of South Africa). 
younger generations have grown up with digital technologies, such as cell phones, computers/laptops and netbooks. They use these for everyday communication, to contact people, to maintain friendships and to build new relationships. Most of the younger generations are constantly connected via social networks and social network tools like Facebook, Instagram and Snapchat (Hartman \& McCambridge 2011:24; Jiang et al. 2010:58). The question, however, is whether a quantity of communication points means meaningful communication.

Originally, the motives for the use of digital devices for communication were inter alia 'escapism ... and surveillance of the world at large'. It changed to more interpersonal communication, because of the need for social interaction, the need for sharing of ideas, the exchange of personal advice, as well as for the expression of care and companionship similar to face-to-face interaction (Dimmick, Kline \& Stafford 2000 in Pearson et al. 2009/2010:45-46). Unfortunately, traditional non-verbal communication does not necessarily play a major role in CMC, which may influence meaning in interpersonal communication (Lo 2008; Venter 2017:498). The premise of this research was that CMC messages often lack adequate non-verbal cues influencing the understanding of emotions and attitudes, thus compromising meaningful communication and personal understanding of the other.

The research question for this literature review was whether communicating with others mainly through digital means without adequate non-verbal cues would influence meaningful interaction between people. The researcher used the cuesfiltered-out approach and the social presence theory with the literature review in order to get some clarity on this question. Social presence refers to the satisfaction of a receiver of online communication. It refers to the receiver's perception of the sender of the online communication as a real person with whom he or she could have a connection or interpersonal relationship in mediated interaction (Alsadoon 2018:227; Lee \& Huang 2018:115-11656). The cues-filtered-out approach emphasises that CMC, without adequate non-verbal cues, may result in conflicts, because of misunderstanding of message content, attitude and emotions (Lo 2008:595; Venter 2017:502). The premise of the author was that face-to-face communication was always important for the creation of meaningful interaction. CMC could add to face-to-face communication to make interaction more recent in the digital world.

\section{Face-to-face versus digital or computer-mediated communication}

Face-to-face communication is the best way to convey emotions and attitude. It gives synchronous feedback between two or more persons and conveys verbal and nonverbal social cues to enhance understanding. The verbal and non-verbal cues help to make decisions and to solve problems between people. Face-to-face interaction is a good way of developing meaningful relations with others
(Trevino, Daft \& Lengel 1990 in Sherblom 2010:499; Gapsiso \& Wilson 2015:208; Venter 2017:502). Verbal and non-verbal communications make use of a 'socially shared symbol system' (Mandal 2014:417). Communication is a process of using symbols for transactional interaction (Ayee 2013:4). Verbal communication uses language as a symbol, but nonverbal communication uses other symbolic activity without emphasis on language (Duck \& McMahan 2009:54-57; Venter 2017:501). Both are important for meaningful interaction. The verbal and non-verbal cues in face-to-face communication give a richness to this kind of interaction. Lamichhane (2016:92) indicates that verbal and non-verbal communications are intertwined, because both are important for face-to-face communication.

Non-verbal communication is especially important for emotional messages where feelings, attitude and intent of senders are made clear (Lamichhane 2016:92). Nagel and Lubinga (2015:88) indicate, for example, the importance of a mere smile or hug when comfort is needed. Even if people do not see each other, their tone of voice can indicate sympathy, empathy or ignorance. Verbal communication is more important for conveying ideas (Lo 2008:595; Venter 2017:502). Verbal communication is also important for 'facilitating human co-operation, enabling us to share assumptions, intentions and communicative conventions, and in so doing regulate social behaviour and human relationships' (Narunsky-Laden 2015:109). It is normally in written format with only emojis or emoticons as an indication of the feelings experienced with the written message.

When verbal and non-verbal messages are in conflict, verbal contents are often disregarded in favour of the non-verbal understanding of the message. Non-verbal communication 'creates comfort in communication by avoiding ambiguity and generating common ground for understanding' (Lamichhane 2016:92-93). It strengthens and explains verbal communication through cues like bodily movement, tone of voice, use of space, time and touch (Duck \& McMahan 2009:63-76; Nagel \& Lubinga 2015:88) to enhance the meaning and understanding of verbal messages. Non-verbal communication enhances understanding between people, because it explains verbal communication, emotional climate, as well as social impressions as expressed by conversation partners (Bente \& Kramer 2011:176; Nagel \& Lubinga 2015:89; Venter 2017:501). Non-verbal interaction includes messages without actual words, which can be either intentional or unintentional (Mandal 2014:417). This kind of communication is often 'beyond' our control. People cannot but communicate, because even if we are silent, our non-verbal communication will tell what we think or feel (Nagel \& Lubinga 2015:89). Non-verbal communication is contextual. Verbal communication mostly conveys content, whereas non-verbal communication conveys more relational information, for example, how a person feels about a matter or another person. For instance, an expression 'nice work' could be a compliment for work well done or a sarcastic remark for work half-done, depending on the context 
(Nagel \& Lubinga 2015:90). It will be difficult to deduce the exact meaning of the above phrase by only reading the comment without being with the person, therefore the emphasis on context. Non-verbal communication can also be culture-specific, for example, the head gestures for 'yes' and 'no' are universal, but personal space between conversation partners varies between cultures (Mandal 2014:419).

People moved from face-to-face communication to mainly using CMC, because of the availability and accessibility of digital communication technologies. The use of traditional ways of communicating has changed, because of social changes and trends, as well as the availability of new digital and social network tools. Person-to-person communication has been transformed to connection via digital tools. Social networks have created a new type of interpersonal communication (Alshenqeeti 2016:56; Gapsiso \& Wilson 2015:203). The younger generations prefer communication through social networks and instant messaging (Heng \& Yazdanifard 2013:838), because of quick and easy access to other people. According to Srivastava (2012:12; Boyle \& O'Sullivan 2016:299; Duck \& McMahan 2009:243), social networking services allow for relationships with people around the globe. It is used to form, maintain or end personal relationships. According to Boyle and O'Sullivan (2016), CMC:

\begin{abstract}
... permits greater access to a person than relying on face-to-face interactions alone, as messages can be transmitted, received and reciprocated near constantly throughout the day and in almost any context. (p. 299)
\end{abstract}

Although CMC is seen as constant, quick and easy communication, it is also seen as a leaner way of communicating in comparison to face-to-face communication, because it conveys limited information, mostly without adequate non-verbal cues. It also gives very little feedback during online conversations (Sherblom 2010:499; Venter 2017:502). The less people communicate face-to-face, the less competent they become in interacting in that way, with the result that the quality of human interactions can be compromised (Gapsiso \& Wilson 2015:217). There is often a lack of meaningful two-way conversation where the conversation partners try to understand the more emotional content of a conversation, because of limited non-verbal cues.

There are, thus, more challenges to CMC than what people would anticipate.

\section{Challenges with digital or computer- mediated communication}

Computer-mediated or digital communication lacks the richness of, for instance, using tone, body movement, gestures or facial expressions to convey messages. The lack of nonverbal cues makes CMC more impersonal in nature. It can lead to loneliness because people often communicate whilst sitting in isolation in front of their digital devices. The lack of non-verbal communication and physical presence may be experienced as a lack of social presence from another human being (Turnbull 2010:6; Venter 2017:502).

It is hard to interpret aspects such as irony and humour in mediated communication, because of the lack of non-verbal cues. It can result in misunderstandings and conflict (Sherblom 2010:478; Venter 2017:502). Because of a lack of face-to-face communication, people often present an idealised version of themselves, thus becoming less inhibited, involving more inappropriate self-disclosure on, for instance, social networks. A discussion about the challenges of mainly using CMC for communication follows.

\section{Lack of non-verbal cues}

Non-verbal language is seen as the best way of conveying emotional and physical cues through, for example, tone, hand and eye gestures or other visual elements (Alshenqeeti 2016:60). The cues-filtered-out approach and social presence theory indicate that mediated communication often lacks non-verbal cues and the physical presence of the sender, which may lead to misunderstandings, resulting in conflict (Krohn 2004:322; Venter 2017:502).The lack of non-verbal cues in mediated messages is problematic when the sender wants to express emotions or attitudes. The social presence theory argues that with more non-verbal cues, the receiver of the message experiences 'more social presence - a sense of the other - ... leading to warmth, friendliness, and satisfaction with the interaction' (Venter 2017:502; Walther 2011:19).

The traditional non-verbal dimensions such as 'facial expression, gestures, body positions, personal distance, vocal variety, and eye contact' are often lost in mediated communication (Krohn 2004:322). People who mostly use digital tools for interaction use emojis or emoticons to convey emotions and attitudes. However, it remains problematic, because it is still verbal cues written as text (Lo 2008:595). Developments such as the use of emoticons and emojis as substitutes for traditional non-verbal communication have changed digital interaction in a positive way (Alshenqeeti 2016:56) to assist in expressing attitudes, emotions and intent:

The wide use of emojis ... across a range of cultures suggest that users consider them as an effective and powerful tool for communicating feelings and emotions in digital medium. (p. 63)

Human communication is, however, complex and emojis can either 'clarify or confuse the receiver, because they indicate presence of emotion but absence of the individual, due to their digital nature', and it expects a level of interpretation 'depending on the focus of the message, the relationship between the interlocutors and the context of the message' (Alshenqeeti 2016:59).

The use of, for instance, Skype or video conferencing also helps to eliminate the negative effect of limited non-verbal cues, because communicators can see and hear each other. 
It gives a sense of presence. Unfortunately, communication through the mentioned media means that communicators normally sit relatively still in front of the two-dimensional display on a screen without perfect resolution. It may influence the fluency of the process (Bitti, Enrico \& Garotti 2011:83-92; Venter 2017:503). There is a lack of, for instance, olfactory and kinaesthetic sensory information in this kind of communication (Parkinson \& Lea 2011:102-109; Venter 2017:503), creating a weaker quality of communication.

Social presence is very important for socio-emotional communication and understanding of another person. Social presence is defined as '... the salience of psychological proximity, immediacy, intimacy, and familiarity experienced with the other person, communication, and relationship' (Yamada 2009 in Sherblom 2010:500; Lowenthal \& Snelson 2017:142; Venter 2017:502). Social presence is important for a communicator to experience the salience ('being there') of an interaction partner. With high social presence, the communicator experiences warm, sociable, personal interaction. CMC is often seen as a less personal way of communicating, because it lacks non-verbal cues and senders often struggle to interpret the 'mood of [the] message', which can cause misunderstandings (Lo 2008:595; Lowenthal \& Snelson 2017:142). CMC may, thus, be better suited for task-orientated communication instead of interpersonal communication (Lowenthal \& Snelson 2017:142), because it restricts the communication of social information about a specific person (Sherblom 2010:500).

\section{Self-disclosure}

Another problem with the use of CMC is the use of selfdisclosure, where people reveal personal information about themselves to other people (Boyle \& O'Sullivan 2016:299). Jiang et al. (2010:58-60) did a literature study on self-disclosure, and the information from various authors indicates that the 'self-revelation of private thoughts, experiences and emotions' is widespread on social media and social networks. Normally it entails information about the self that is normally kept hidden from most people. Noska, Wood and Molema (2010 in Jiang et al. 2010:59) did a content analysis of Facebook profiles and found that the average Facebook user discloses approximately $25 \%$ of highly personal, sensitive and potentially stigmatising information (e.g. political views, sexual orientation, religious affiliation and personal phone numbers).

Too much self-disclosure is seen as problematic, because people do not discern between receivers of these kinds of personal and intimate knowledge. The disclosure of personal, intimate information about the self fosters reciprocation and closeness in relationships, often with total strangers. Selfdisclosure happens more often in CMC than in face-to-face communication, maybe because of perceived anonymity. The absence of non-verbal cues where the sender of the message cannot see the reaction of the receiver makes it easier to disclose private information. Self-disclosure combined with personal questions from others seem to be strategies for getting to know another person better. Relationships develop quickly in this way, notwithstanding its superficiality (Boyle \& O'Sullivan 2016:300).

People often optimise their self-impression on social media or in e-mail messages, because they have time to think it through; therefore, they use selective self-presentation and self-disclosure to portray the best image of themselves to attract others to them (Jiang et al. 2010):

Receivers of CMC messages tend to over interpret limited socioemotional and social identity cues available in text-based interaction leading to intensified impressions of the sender's personal qualities and their relationships. (p. 61)

Receivers of CMC communication must be cautious of the interpretation of self-disclosure and self-impression in messages without non-verbal cues. Social networking sites changed the way people think about self-disclosure. Perceptions of social value and belongingness are often tied to the number of 'friends' on the social networking sites a person subscribes to. Although social worth was always connected to friends, the quantity seems to be more important on social networks than the quality of friends and communication (Duck \& McMahan 2009:244).

\section{De-individuation}

The de-individuation theory (Lea \& Spears 1991 in Jiang et al. 2010:63) indicates that 'stereotyped and exaggerated partner impressions occur in CMC because of disproportionate reliance on the minimal identity cues available in textbased interactions'. Lee (2004:234-235) concurs with the above notion of the effects of social de-individuation, mentioning group salience and group conformity as effects of the lack of individuating information in CMC. Individuals often depersonalise themselves when interacting with others, because of visual anonymity, thus becoming more susceptible to group influence. The individual is not important in this theory, because of group stereotyping. According to Sherblom's (2010:500-501; Venter 2017:503) research, communicators in CMC often optimise their selfpresentation for socially desirable relationships. They use group identity cues of what is acceptable regarding gender, physical appearance and ability to conform to group norms. A desirable self-image is constructed by editing, reviewing and constructing online message content to attract others. All of the above means the reduction of inhibitions and the facilitation of indiscriminate self-disclosure. Over-sharing of information in a non-normative way may demonstrate a lack of inhibition leading to de-individuation where the group becomes the norm (Cooke 2016:253). Group membership is often the driving force in online relationships. Unfortunately, when group membership becomes more important than personal, individual identity, people see themselves more as 'part of a group than as individuals' (Wang, Walther \& Hancock 2009:60-61). 


\section{Meaningful interpersonal communication}

Face-to-face interaction uses active, passive and interactive strategies, which add quality to communication. Communicators use verbal and non-verbal communications to express their feelings and attitudes. Other people get to know them and see them as persons with real needs and emotions. $\mathrm{CMC}$, on the other hand, uses mainly interactive strategies. Communicators using digital tools get to know others by asking questions and listening for self-disclosure. They cannot really observe or compare receivers of their communication; neither can they create situations to see how people react. Communicators using $\mathrm{CMC}$ can use resources like internet profiling and electronic searches whilst communicating online (Sherblom 2010:503) to get to know another person better. It is easier to communicate via CMC. Access and availability makes it the preferred mode of interaction for many people, because face-to-face communication is not always feasible in the fastmoving digital world. The challenge, however, remains that the information is mostly in written format, which can be manipulated by the portrayed person to create an acceptable image. The non-verbal communication happens through emojis and emoticons or on a screen. Social presence is not important in $\mathrm{CMC}$; therefore, meaningful interaction could be compromised.

Quality interpersonal communication involves people listening to each other with concern and empathy. Communicators should be able to express their emotions in a way that conversations are meaningful for both the sender of the message and the receiver. Individuals should be able to communicate with confidence indicating that they know and accept themselves instead of merely adhering to group norms (Drago 2015:14; Sherblom 2010:504; Venter 2017:502). Face-to-face communication would be more suitable for the above requirements, but seeing that it is not always possible in the digital world, people must be cautious of only using CMC. A combination of face-to-face communication and $\mathrm{CMC}$ would be the answer for meaningful communication and will be a more recent way of communicating in a world where digital tools and social networks are becoming the norm for interaction.

\section{Conclusion}

Human beings often take their ability to communicate and to share meaning with each other for granted. Interpersonal communication indicates a distinctively human activity. Human beings communicate differently from, for instance, animals - they share, give and inform through communication (Chasi 2015:5). Tomasello (2009, 2010 in Chasi 2015:5) indicates that the mentioned attributes enable people to communicate with symbols and signs, helping to create trust to establish common ground for meaningful communication. Burger (2015:37) indicates that a 'meaningcentred approach to communication' means that two or more people express how they see a point or feel about an issue until they reach a 'shared meaning' or an understanding of each other. It is important for meaningful interaction to give and receive feedback.

Human beings are in essence social beings. They need to be connected to others to experience a meaningful existence. CMC and face-to-face communication assist with the feeling of connectedness. Social presence with non-verbal and verbal cues is, however, important for understanding each other. The author is not sure that without social presence an authentic connection is possible in the end. If a person sits by himself or herself talking online, feelings of isolation and loneliness might be a result, taking an important aspect of being authentically human away from the person. Online communication often lacks warmth, social presence and feelings of belonging.

The use of mainly CMC, because of the rapid development of technology, may cause people to be so immersed in the 'digital world' that they may not be 'present enough in the real world'. Sometimes, people struggle to differentiate between their real self and their digital self, because of the emphasis on acceptance within specific groups on social networks. Face-to-face time with others are slowly changing to screen-to-screen time that may influence getting to know people as real persons (Drago 2015:13). Drago (2015:14) referred to the research conducted by Przybylski and Weinstein (2012) indicating the negative influence of mainly communicating through CMC on 'closeness, connection, and conversation quality'. CMC may diminish people's ability to communicate face-to-face if that is their preferred way of communicating. Getting to know the self and others as individuals may be an issue in mainly using CMC. Combining CMC with face-to-face communication might be a better way of interaction between human beings where emotions, feelings, attitude and intent are just, if not more, important than the verbal or written interaction of CMC.

\section{Acknowledgements}

The author guarantees that the contribution is original, has not been published previously and is not under consideration for publication elsewhere. It will not be submitted to another journal before a final decision from HTS. This article was presented as part of the 2018 SASRF seminar on 'What it means to be human in a technological era' from an educational psychology perspective, therefore contributing to the interdisciplinary nature of the SASRF research project.

\section{Competing interests}

The author declares that she has no financial or personal relationship(s) that may have inappropriately influenced her in writing this article.

\section{Disclaimer}

The views and opinions expressed in this article are those of the author and do not necessarily reflect the official policy or position of any affiliated agency of the author. 


\section{References}

Alsadoon, E., 2018, 'The impact of social presence on learners' satisfaction in mobile learning', The Turkish Online Journal of Educational Technology 17(1), 226-233.

Alshenqeeti, H., 2016, 'Are emojis creating a new or old visual language for new generations? A socio-semiotic study', Advances in Language and Literary Studies $7(6), 56-69$.

Ayee, E.S.A., 2013, 'Human communication revisited - A biblical perspective', Koers 78(1), 1-16. https://doi.org/10.4102/koers.v78i1.549

Bente, G. \& Kramer, N.C., 2011, 'Virtual gestures: Embodiment and nonverbal behaviour in computer-mediated communication', in A. Kappas \& N.C. Kramer (eds.), Face-to-face communication over the internet - Emotions in a web culture, language and technology, pp. 176-210, Cambridge University Press, culture, langu York.

Bitti, P., Enrico, R. \& Garotti, P.L., 2011, 'Nonverbal communication and cultural differences: Issues for face-to-face communication over the internet', in A. Kappas \& N.C. Kramer (eds.), Face-to-face communication over the internet - Emotions in a web culture, language and technology, pp. 81-99, Cambridge University Press,
a a web culture,
New York.

Boyle, A.M. \& O'Sullivan, L.F., 2016, 'Staying connected: Computer-mediated and face-to-face communication in college students' dating relationships', Cyberpsychology, Behavior, and Social Networking 19(5), 299-307. https://doi. Cyberpsychology, Behavior, and
org/10.1089/cyber.2015.0293

Burger, M., 2015, 'Conceptualising communication: Theory and method', in S. Steinberg \& G. Angelopulo (eds.), Introduction to communication science for Southern African students, pp. 24-47, Juta, Cape Town.

Chasi, C., 2015, 'A brief introduction to human communication', in S. Steinberg \& $\mathrm{G}$. Angelopulo (eds.), Introduction to communication science for Southern African students, pp. 3-23, Juta, Cape Town.

Cooke, N.A., 2016, 'Information sharing, community development, and deindividuation in the e-learning domain', Online Learning 20(2), 244-260. https://doi.org/ 10.24059/olj.v20i2.614

Drago, E., 2015, 'The effect of technology on face-to-face communication', The Elon Journal of Undergraduate Research in Communications 6(1), 13-19.

Duck, S. \& McMahan, D.T., 2009, The basics of communication - A relational perspective, Sage, Los Angeles, CA.

Gapsiso, N.D. \& Wilson, J., 2015, 'The impact of the internet on teenagers' face-toface communication', Journal of Social Sciences 13(2), 202-220.

Hartman, J.L. \& McCambridge, J., 2011, 'Optimizing millennials' communication styles', Business Communication Quarterly 74(1), 22-44. https://doi.org/10.1177/ 1080569910395564

Heng, C.Y. \& Yazdanifard, R., 2013, 'Generation gap: Is there any solid solution? From human relation point of view', International Journal of Economy, Management and Social Sciences 2(10), 837-840.

Jiang, L.C., Bazarova, N.N. \& Hancock, J.T., 2010, 'The disclosure-intimacy link in computer-mediated communication: An attributional extension of the hyperpersonal model', Human Communication Research 37, 58-77. https://doi. org/10.1111/j.1468-2958.2010.01393.x

Krohn, F.B., 2004, 'A generational approach to using emoticons as nonverbal communication', Journal of Technical Writing and Communication 34(4), 321-328. communication', Journal of Technical Writing and Com
https://doi.org/10.2190/9EQH-DE81-CWG1-QLL9

Lamichhane, Y.R., 2016, 'Non-verbal skills: Unavoidable in communication', Repositioning 1(1), 91-98. https://doi.org/10.3126/repos.v1i0.16046
Lee, E., 2004, 'Effects of visual representation on social influence in computermediated communication - Experimental tests of the Social Identity Model of deindividuation effects', Human Communication Research 30(2), 234-259. deindividuation effects', Human Comm
https://doi.org/10.1093/hcr/30.2.234

Lee, S.J. \& Huang, K., 2018, 'Online interactions and social presence in online learning', Journal of Interactive Learning Research 29(1), 113-128.

Lo, S., 2008, 'The nonverbal communication functions of emoticons in computermediated communication', Cyber Psychology \& Behavior 11(5), 595-597. https:// doi.org/10.1089/cpb.2007.0132

Lowenthal, P.R. \& Snelson, C., 2017, 'In search of a better understanding of social presence: An investigation into how researchers define social presence', Distance Education 38(2), 141-159. https://doi.org/10.1080/01587919/2017.1324727

Mandal, F.B., 2014, 'Nonverbal communication in humans', Journal of Human Behavior in the Social Environment 24(4), 417-421. https://doi.org/10.1080/1091 1359.2013.831288

Nagel, P. \& Lubinga, E., 2015, 'Nonverbal communication', in S. Steinberg \& G. Angelopulo (eds.), Introduction to communication science for Southern African students, pp. 85-106, Juta, Cape Town, South Africa.

Narunsky-Laden, S., 2015, 'Language and communication', in S. Steinberg \& $\mathrm{G}$. Angelopulo (eds.), Introduction to communication science for Southern African students, pp. 107-138, Juta, Cape Town, South Africa.

Parkinson, B. \& Lea, M., 2011, 'Video-linking emotions', in A. Kappas \& N.C. Kramer (eds.), Face-to-face communication over the internet - Emotions in a web culture, language and technology, pp. 100-126, Cambridge University Press, New York.

Pearson, J.C., Carmon, A., Tobola, C. \& Fowler, M., 2009/2010, 'Motives for communication: Why the millennial generation uses electronic devices', Journal of the Communication, Speech \& Theatre Association of North Dakota 22, 45-56.

Przybylski, A.K. \& Weinstein, N., 2012, 'Can you connect with me now? How the presence of mobile communication technology influences face-to-face conversation quality', Journal of Social and Personal Relationships, 1-10.

Sherblom, J.C., 2010, 'The computer-mediated communication (CMC) classroom: A challenge of medium, presence, interaction, identity, and relationship' Communication Education 59(4), 497-523. https://doi.org/10.1080/03634523. 2010.486440

Solomon, D. \& Theiss, J., 2013, Interpersonal communication - Putting theory into practice, Routledge (Taylor \& Francis Group), New York.

Srivastava, P., 2012, 'Social networking and its impact on education-system in contemporary era', International Journal of Information Technology Infrastructure $1(2), 11-18$

Turnbull, C.F., 2010, 'Mom just facebooked me and Dad knows how to text: The influences of computer-mediated communication on interpersonal communication and differences through generations', Elon Journal of Undergraduate Research in Communications (Elon University) 1(1), 5-16.

Venter, E., 2017, 'Bridging the communication gap between generation $Y$ and the baby boomer generation', International Journal of Adolescence and Youth 22(4), 497-507. https://doi.org/10.1080/02673843.2016.1267022

Walther, J.B., 2011, 'Visual cues in computer-mediated communication: Sometimes less is more', in A., Kappas \& N.C. Kramer (eds.), Face-to-face communication over the internet - Emotions in a web culture, language and technology, pp. 17-38, Cambridge University Press, New York.

Wang, Z., Walther, J.B. \& Hancock, J.T., 2009, 'Social identification and interpersonal communication in computer-mediated communication: What you do versus who you are in virtual groups', Human Communication Research 35, 59-85. https:// doi.org/10.1111/j.1468-2958.2008.01338.x 